\title{
Research on Augmented Reality Experience Design of Tujia Brocade Products Based on Emotion Cognition
}

\author{
Ning $\operatorname{Yun}^{1 *}$ \\ ${ }^{1}$ Guangxi Vocational and Technical College, Nanning, Guangxi, China
}

\begin{abstract}
Augmented reality technology is an extension of virtual reality. Voice input and output devices, image input and image display devices, touch-tap gesture action input and visual tracking devices are used in various computer systems to improve computer efficiency and human-computer interaction accuracy, which can improve the interactivity and user experience of augmented reality systems. Augmented reality technology superimposes the virtual world and the real world, and its final interactive operation has an enhanced effect on the senses of the participants. Augmented reality has been able to be used more maturely in many fields, and its use in the art field has become a new direction for designers to explore art and practice. This study explores the user's emotional perception process of Tujia brocade products from the theoretical level, constructs an augmented reality experience model applied to Tujia brocade, and proposes a design process for the implementation of augmented reality.
\end{abstract}

\section{Introduction}

Augmented reality technology incorporates a variety of technologies such as digital image processing, computer vision, pattern recognition and human-computer interaction. Based on the complexity of augmented reality technology, there are still many technical difficulties that have not been completely overcome [1]. The emerging $5 \mathrm{G}$ network, which provides mobile devices with less latency, better connectivity and higher data throughput, has laid the foundation for the development of augmented reality systems on mobile devices. Augmented reality, as a new technology in the field of computing, is becoming more and more widely used [2]. As a product of intangible cultural heritage Tujia brocade weaving skills, Tujia brocade products have profound cultural heritage and historical value, however, due to the influence of foreign cultural invasion and impact of new technology products. It has led to its narrow audience, low economic benefits and insufficient competitiveness, and Tujia brocade products are declining [3]. As far as the sharing and dissemination of intangible cultural heritage is concerned, the introduction of new technologies to realize surreal and holographic digital resource experiences of intangible cultural heritage represents the direction of future development and will gradually develop into an important means to assist in the act of conservation and inheritance. In order to make the value of Tujia brocade easily and quickly perceived by users, this paper explores the relationship between users' emotions and the value of Tujia brocade products by using augmented reality technology and from the perspective of emotional perception, helping users to perceive the value of Tujia brocade products, in order to play a positive role in preserving and promoting Tujia brocade, and to serve as a reference for other ethnic cultural products facing the same problem, and to promote and We hope to play a positive role in the preservation and promotion of Tujia brocade products, to serve as a reference for other ethnic cultural products facing the same problems, and to help promote and protect traditional cultural diversity.

\section{Basic Concepts of Emotional Cognition}

Cognitive psychology is the science of studying emotions and a very important branch of psychology. In psychology, the purpose of the theory of emotional cognition is that emotions originate from people's evaluation of the things they are stimulated, and the nature of emotions is determined by people's cognitive processes. Emotional psychology is mainly the study of emotional changes such as joy and sadness, expectation and disappointment, enthusiasm and indifference, anger and panic, worry and anxiety [4]. Emotions and emotions are the corresponding attitude experiences and behavioral responses produced by people after they perceive objective things. They usually consist of subjective experience, external performance, and physical arousal. The essence of emotion is emotion, and the appearance of emotion is emotion.

The basic process of emotion generation is: stimulating situation-evaluation-emotion. This shows that the generation of human emotions depends on people's perception of stimuli, not caused by objective emotional stimuli [5]. People's cognitive process of external things

*Corresponding author's e-mail: 17280319@nytdc.edu.cn 
is a process from cognition to intention, that is, "cognitionevaluation-emotion-need-thinking-intention". Cognition consists of perception, attention, memory, reasoning, decision-making, etc. Composed of inner activities, the functions of these activities together construct a complex psychological system, and its comprehensive manifestation is cognition [6].

In the 1960s, theories about the information processing structure of the brain emerged. Lindsay and Norman established an emotional arousal model based on the process of emotional arousal, as shown in Figure 1. They believe that emotion is the result of the combined effect of cognitive processing and neural activation [7].

If there is a certain degree of difference between the result and the expected situation, such as a sudden accident, the cognitive comparator will generate a corresponding signal to stimulate the nerve, perform chemical activation, stimulate the brain, and change to the corresponding activation form, and then produce mood. The emotion generation model is shown in Figure 2.

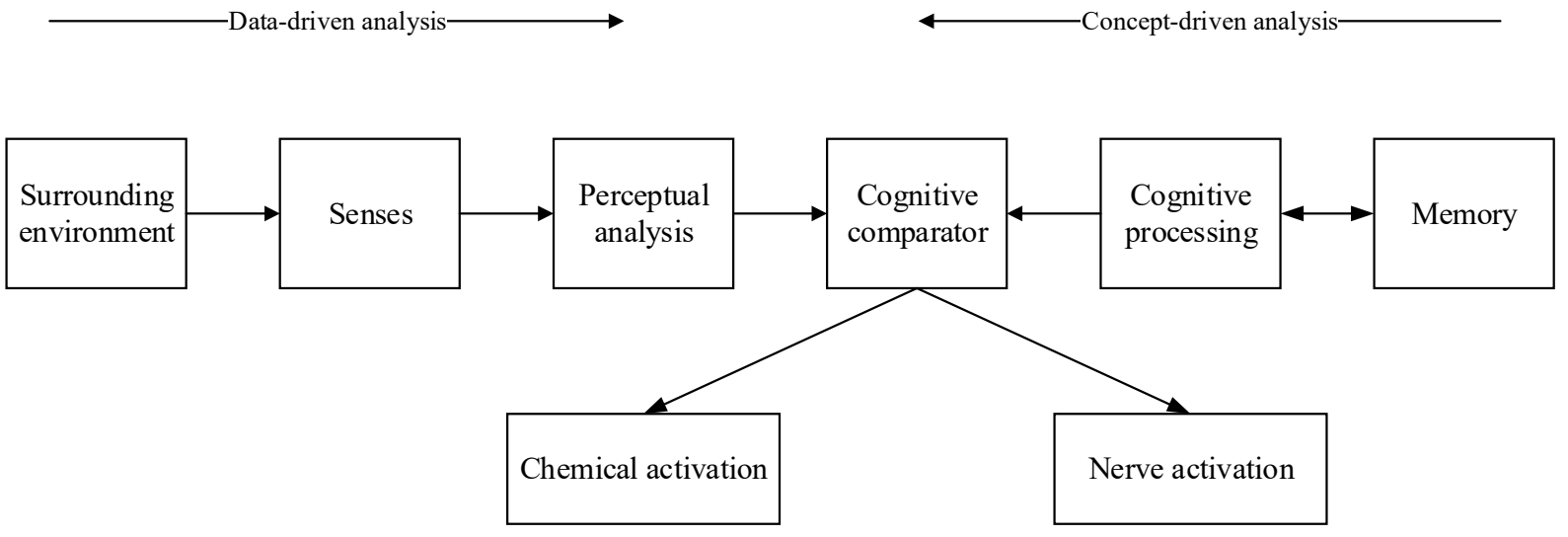

Figure 1. Emotional arousal model

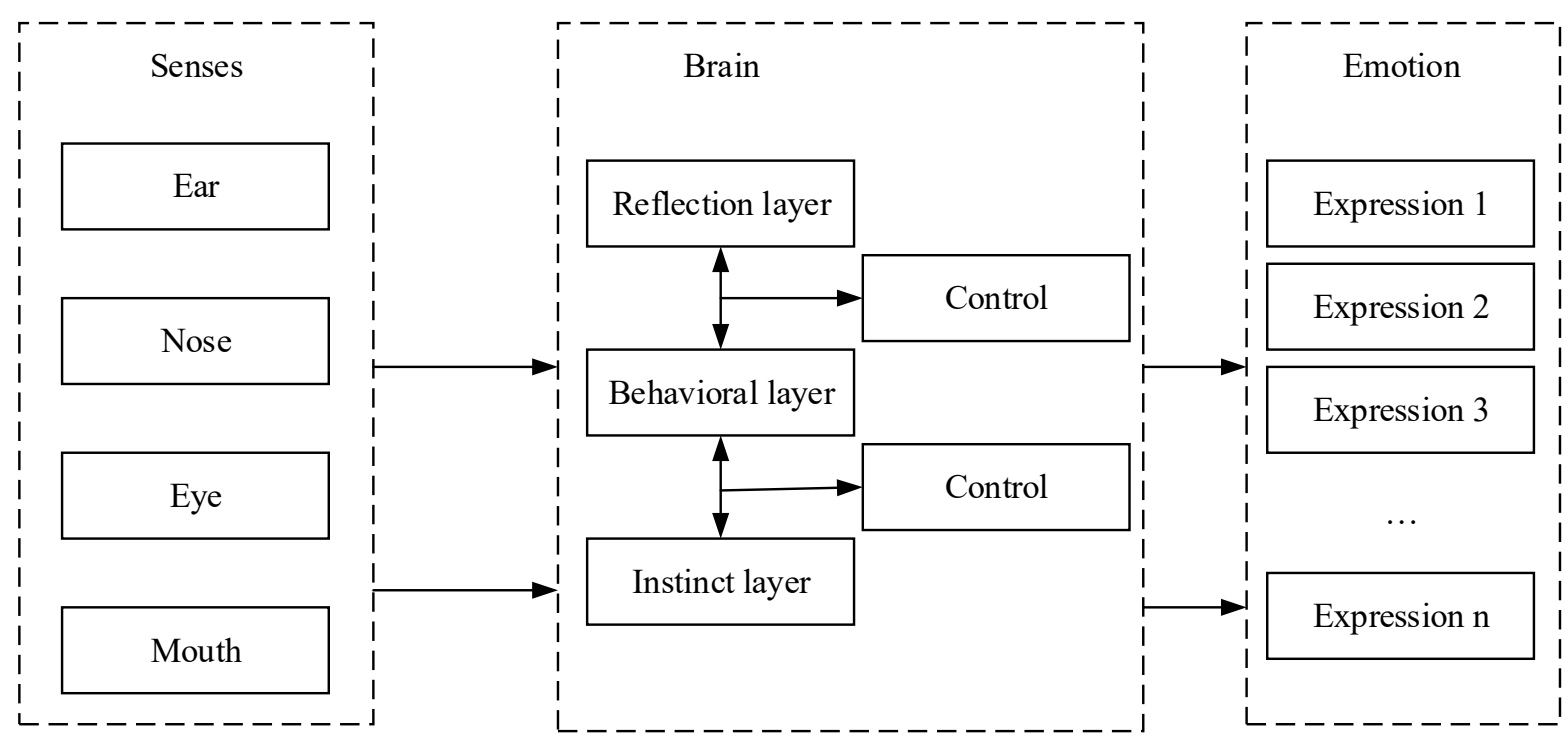

Figure 2. Emotional patterns

\section{Exploration of key elements of Tujia brocade product value recognition}

The Tujia cultural elements contained in Tujia brocade products give it a unique charm that is different from ordinary brocade products. Its value is not only in its formal characteristics, but also in its inherent value, so it is necessary Analyze its value factor value [8].

There is a cognitive process in the process of users experiencing cultural products. After the user physically perceives cultural products, the user will enter a judgment process, and the judgment result will affect the user's emotion generation and the end user's expression. The attitude towards cultural products is the process of "physiological perception - judgment - emotional expression". The specific user's emotional perception of cultural products includes the following parts, as shown in Figure 3. 


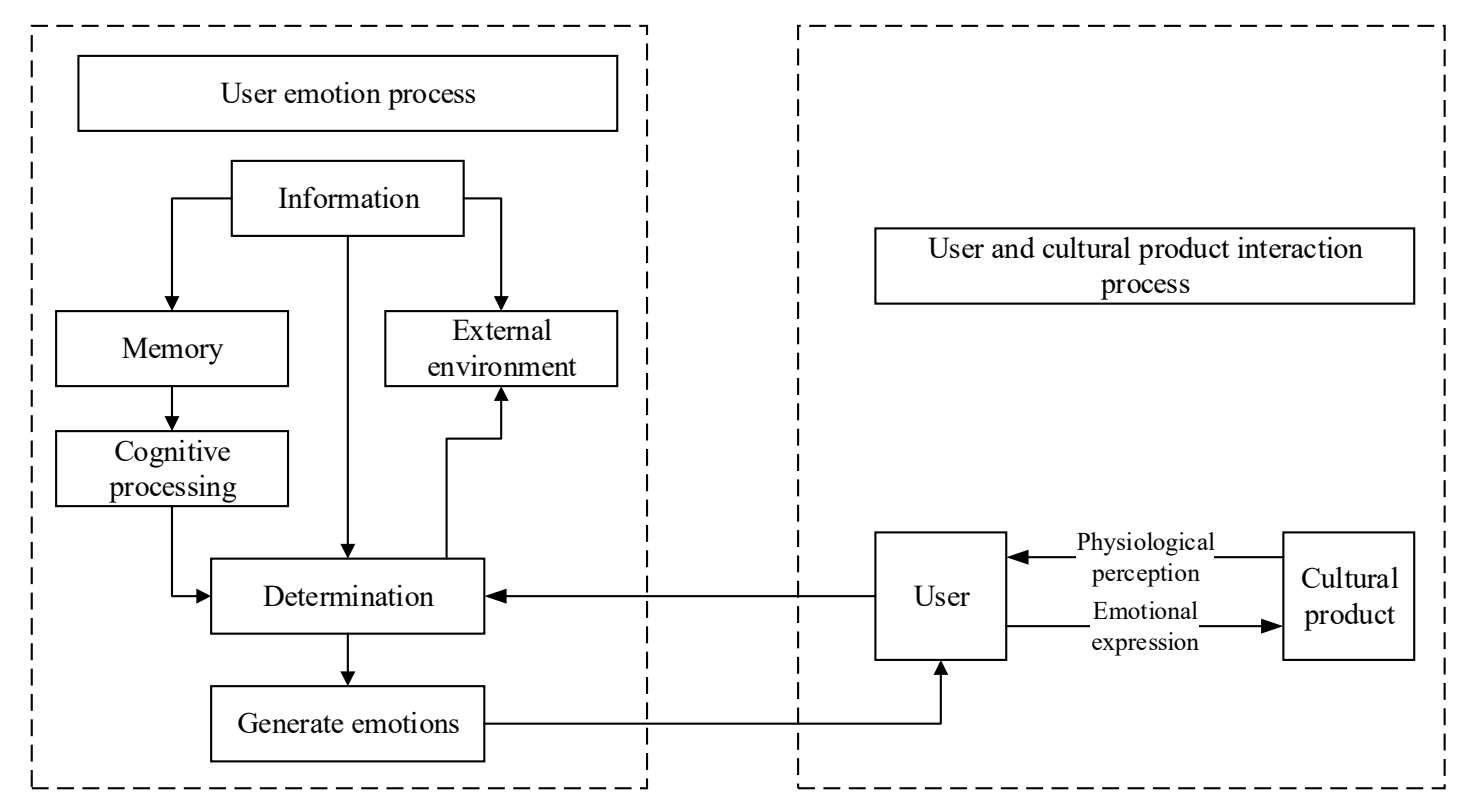

Figure 3. User emotion recognition process of cultural products

\section{Build an augmented reality experience model and design process for Tujia brocade products}

\subsection{Constructing an augmented reality experience model of Tujia brocade products}

The purpose of constructing the augmented reality experience model of Tujia brocade products is to clarify the feasibility of applying augmented reality to Tujia brocade products and provide theoretical support for subsequent design practices. The augmented reality applied to Tujia brocade products should meet the users' cognitive and emotional needs for Tujia brocade. These two parts require users to be able to perceive through the senses and physiology. The existing augmented reality manifestations are mainly the display of the visual layer [9]. Text, pictures, virtual models and other forms to display content, users perceive the content with their senses to meet their needs. The extracted key elements of Tujia brocade product value recognition do not exist objectively. Therefore, the cognitive elements of Tujia brocade product value need to be converted into userperceivable content before the subsequent theoretical model construction and design plan can be carried out.

The cognitive elements of Tujia brocade value transform the content that adults can perceive, including the name and origin of brocade products, the production process of brocade products, production materials, production machines, influence regions, outstanding deeds, prices and sharing, etc., but the content is complicated and needs to be sorted out to facilitate subsequent design practice. The name and the cultural heritage derived from it, it is the basic information of an item; the production process, production materials, and production machines are derived from the production process. These three points can basically reflect the situation of a process, so it can be called a process display; influencing areas, excellent deeds and prices, it shows the development trajectory of a product from the three aspects of influence, achievement and economic factors, so it can be called the current development; sharing comes from the role of social interaction [10]. The core of social interaction is the communication between people. The word sharing can well reflect this, so it can remain unchanged. After finishing the above content, the details are shown in Table 1.

After the user recognizes the Tujia brocade pattern as the recognition target through augmented reality, the augmented reality will display the content corresponding to the display elements. During the user's interaction with augmented reality, the user's experience needs are met. At the same time, the user will perform the emotional process of "perception-judgment-emotion" to determine whether the user's value perception and emotional needs are met. Finally, the user's emotions are evaluated as a basis for judging whether augmented reality can achieve the purpose of "improving users' awareness of the value of Tujia brocade products", as shown in Figure 4.

Table 1. Perceivable transformation of the cognitive elements of the value of Tujia brocade

\begin{tabular}{lll}
\hline Value perception & Specific contents & Display elements \\
\hline Cultural heritage & Origin of the name & Basic Information \\
Commodity attributes & Price & Current development \\
Social function & Screenshot save & Share it \\
Craftsmanship & Process material & Craft show
\end{tabular}




\begin{tabular}{lll} 
Social influence & Affected area & Current development \\
Collection value & Comprehensive influence & Comprehensive influence \\
\hline
\end{tabular}

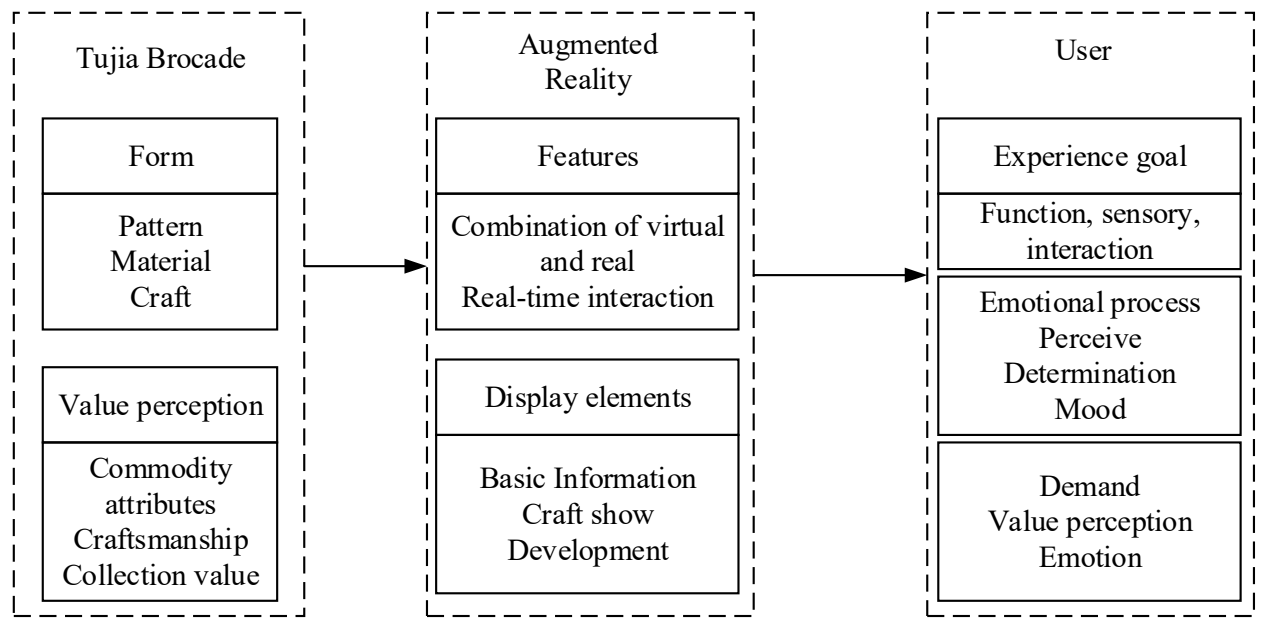

Figure 4. The Augmented Reality Experience Theory Model of Tujia Brocade

\subsection{Augmented reality design process of Tujia brocade products}

According to the experience theory model analyzed above, the design process is shown in Figure 5. First, collect the pattern feature data of Tujia brocade, and the specific content of the brocade name, origin, production material, production machine, production process, influence area, price, and outstanding deeds corresponding to the pattern feature. Through text, pictures, Expressions such as videos are used as content to satisfy the user's sensory experience. Secondly, the augmented reality prototype architecture includes functional prototypes and UI construction. The functional prototype setting mainly reflects the content that satisfies the user's functional experience. The UI construction mainly sets the user interface and interaction methods as the content to satisfy the user's interactive experience.

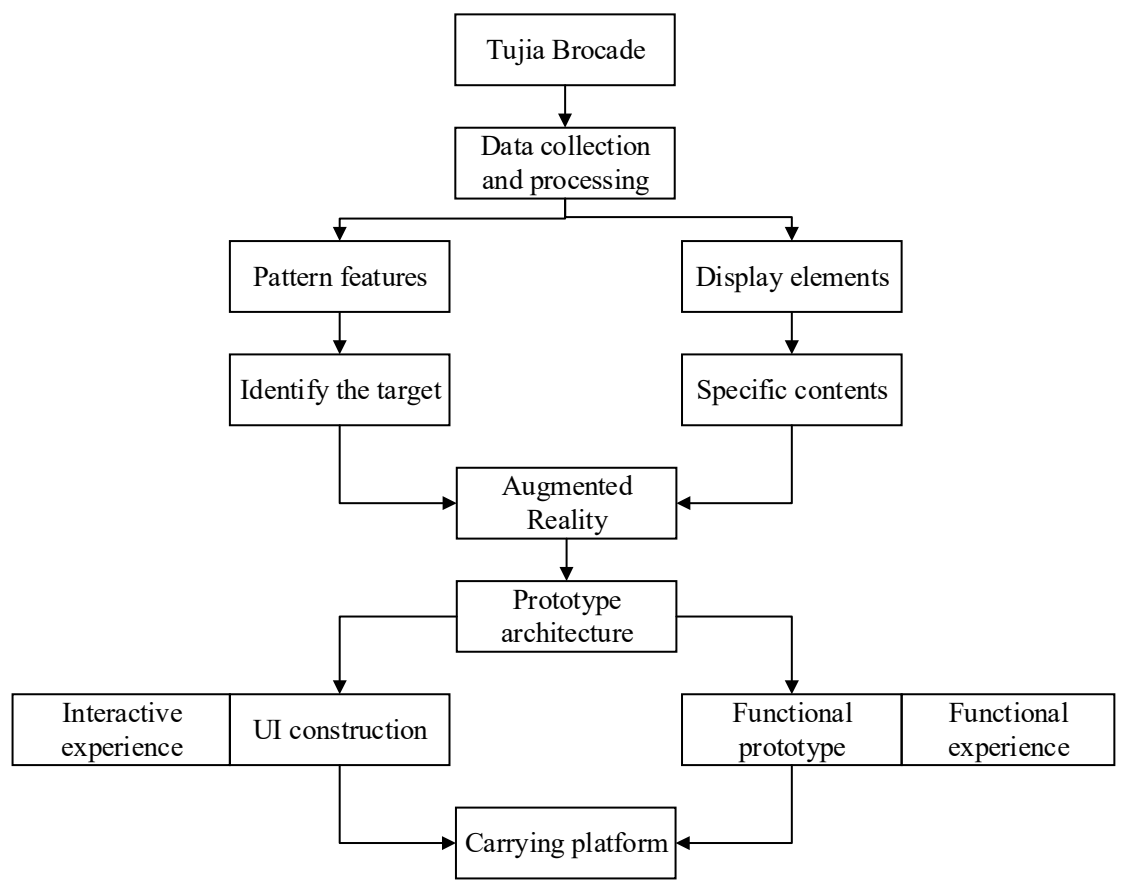

Figure 5. Design process

\section{Conclusion}

Aiming at the current predicament of Tujia brocade products, which makes more people understand and appreciate Tujia brocade products, this article has done a series of investigations. Starting from emotional cognition, the process of user cognitive experience of Tujia brocade is analyzed, the design space of augmented reality applied to Tujia brocade products is explored, the plan is designed, and the augmented reality design program applied to Tujia 
brocade products is completed. In the development of the new era, people's demand for beauty is getting higher and higher, and there is a certain gap between the inheritance and protection of traditional culture and foreign countries, and there are unreasonable places. This article discusses the application of augmented reality technology to Tujia brocade products to generate new development directions and models to enhance the artistic experience of traditional culture. Art works with emotional interaction can establish an emotional connection with the audience. This emotional participation and interaction can arouse people's emotional cognition. There are relatively few domestic studies on the use of augmented reality technology combined with the expression of Tujia brocade products. This kind of art the way is very novel. Due to time and energy constraints, this article did not implement the designed scheme, and the scheme will be implemented later.

\section{References}

1. Li, C., Yang, X.Y., Li, Y. (2020) An Investigation and Analysis of Contemporary Young College Students' Cognition of Filial Piety. Journal of Southeast University (Philosophy and Social Sciences Edition), 22(S2):11-14.

2. Yang, C.Z. (2020) Study on the influence of information flow and emotional granularity on users' cognitive bias in network emergencies. Information Theory and Practice, 43(12):133-143+132.

3. Yao, H.Y., Zhang, J.S., Yan, X.X. (2020) The impact of environmental cognition and environmental emotion on green mining. Journal of Xi'an University of Science and Technology, 40(03):549-556.

4. Fan, Y.H. (2020) Analysis of the calculation model of artificial intelligence emotional cognitive reasoning. Journal of Shanghai Normal University (Philosophy and Social Sciences Edition), 49(02):94-103.

5. Li, C., Zhang, B.C., Wang, Y.Q., et al. (2020) Study on the front face design of baby carriage based on emotional cognition. Packaging Engineering, 41(06):211-217.

6. Zhou, M.X., Wang, R., (2020) The Application Research of Li Nationality Brocade in Tourism Product Packaging Design. Design, 33(21):41-43.

7. Zou, L., Li, H.B., (2020) Research on the Digital Protection Method of Tujia Brocade Pattern. Clothing Designer, (09):68-73.

8. Zhang, C.K, Lin, L., Wen, L.F., et al. (2020) Implementation of an interactive advertising system based on augmented reality. Computer Knowledge and Technology, 16(36):80-83.

9. Liu, Y.Z., Liang, Y., (2020) Cross-media expression of poster design under augmented reality technology. New Media Research, 6(21):119-121.

10. Zhang, W.L., Hu, Y., (2020) Design of Children's Safety Educational Game Based on Augmented Reality Technology. Design, 33(15):150-152. 